\title{
Mechanical Evaluation of Collapse Risk for Osteonecrosis of Femoral Head Post-Medical Treatment
}

\author{
Yuqian Mei \\ Beijing Institute of Technology \\ Qi Tang \\ Beijing Institute of Technology \\ Songhao Chen \\ Beijing Institute of Technology \\ Duanduan Chen ( $\nabla$ duanduan@bit.edu.cn ) \\ Beijing Institute of Technology
}

\section{Research Article}

Keywords: Osteonecrosis of femoral head, collapse risk, finite element analysis, volume ratio, stress index.

Posted Date: January 27th, 2021

DOI: https://doi.org/10.21203/rs.3.rs-138877/v1

License: @ (i) This work is licensed under a Creative Commons Attribution 4.0 International License. Read Full License 


\section{Abstract}

Background: Early-stage osteonecrosis of the femoral head can progressively induce the bone collapse and the accuracy of the collapse risk evaluation is limited. This study aims to propose a potential collapse indicator by biomechanical evaluating the treatment effectiveness.

Methods: Six patient-specific models pre- (initial presentation) and post- medical treatment were established and elastic properties of the bone were assigned with a function of spatial-variant Hounsfield unit values. Morphological and mechanical analyses were compared between pre- and post-treatment models.

Results: In the morphological study, four cases show the reduced ratio of low-density necrotic volume, but not consistent with one-year follow-up results. In the mechanical analysis, the equivalent stress variation near the Ward triangle shows consistency in the one-year outcome. Moreover, a parameter - relative stress index, the ratio between the mean volume stress index and the mean volume equivalent stress, is proposed. A smaller value of the relative stress index indicates stronger bone compressive strength and its variation is consistent with the follow-up results.

Conclusions: Results implicate that to evaluate the effectiveness of medical treatments, the morphologic analysis should be considered but the mechanical capability and the loading transfer path through the necrotic and viable bone play a more important role.

\section{Introduction}

Osteonecrosis of the femoral head (ONFH) is a disabling orthopaedic disease which could lead to progressive destruction of the hip joint and it primarily affects young and middle-aged populations $[13,18]$. It initially occurs due to the reduced blood perfusion inside the femoral head [51], and this precarious blood supply induces cellular death in the bone structure and subsequently necrosis, which modifies the mechanical loading distribution over the proximal femur resulting in impaired bone functions [16]. Several classification systems have been proposed to depict the clinical and radiological severity/progression of ONFH and the ARCO system is the most extensively used [45]. Normally, medical hip-preserving treatments should be performed timely for the early-stage ONFH (ARCO stage I-II) cases, including pharmacological treatment and joint-preserving procedures; while the total hip replacement (THR) has to be considered when the osteonecrosis deteriorated to ARCO stage III -IV. In general, a hip replacement is unable to last the whole lifetime of patients, especially for young adults, normally accompanied with a high revision rate $[19,26,39]$. Therefore, when feasible, attempts for saving the femoral head should be comprehensively considered prior to collapse with use of less invasive treatment modalities. To prevent the progression to late-stage $\mathrm{ONFH}$, the effective initial medical treatment of early-stage ONFH is rather important, improving of the viable bone structure and function so that to reduce the necessity of THR. However, how to accurately assess the effectiveness of the early conservative treatment remains uncertain.

Clinically, medical scans provide evidences for physicians to diagnose the ONFH and assess the treatment effects. On basis of these images, morphological investigations regarding the bone structure during medical treatment, among others, include radian sum, percentage of the proximal sclerotic rim and necrosis index $[9,25,55,53]$. Besides, from the mechanical perspective, patient-specific computational models with detailed bone structure has been extensively used, which is able to give quantitative mechanical information for mechanism analysis of pathological inception and development. In the studies related to ONFH, the effect of surgical operations, i.e. joint-preserving procedures [4-6, 8, 24, $35,38,44,58]$ and THR with specific artificial devices $[7,10,14,34,41]$, is the main concern. However, there is few research paying attention to the pathological development of ONFH and the role of conservative medical treatment at pre-collapse stages.

In the current study, we investigate the mechanical distribution and the corresponding morphological variation of ONFH (ACRO stage II) pre- and post- conservative medical treatment. Computational models were established based on the CT 
scanning of the enrolled six patients with collapse and non-collapse outcomes after treatment. Through the comparative analysis, a novel mechanical parameter is proposed which could be applied in evaluating the modification of bone strength for ONFH. This study aims to provide an objective evaluation method for conservative medical treatment of ONFH and further help the therapy planning.

\section{Methods}

\subsection{Image acquisition and model reconstruction}

Approval of the local ethic committee by China Academy of Chinese Medical Science (WJEC-KT-2016-008-P002) and informed consent from the examined patients were obtained prior to the simulation study. We declare that all methods were performed in accordance with the relevant guidelines and regulations.

Between January 2014 and December 2016, we collected data of six patients with ACRO stage-II ONFH on one single side diagnosed by plain radiographs and MRI according to the consensus on the ONFH diagnosis proposed in 2012 [57]. Apart from this, the enrollment criteria include: (i) at least one-year follow-up time; (ii) the initial CT images clearly present a definite sclerotic boundary; (iii) ages of patients are older than 18. Patients were excluded according to (i) post-traumatic osteonecrosis; (ii) congenital hip disease; (iii) a history of operation on the affected leg. The diseased side and the contralateral healthy proximal femur underwent CT scan (GE Medical Systems, USA) with parameters of 120KV, 300MA, reconstructed slice thickness of $1 \mathrm{~mm}$ and reconstruction increment of $0.625 \mathrm{~mm}$. All patients selected have received consecutive traditional Chinese medicine herbal medication (Jianpihuogu Formula)[23] but without surgical intervention, which includes oral decoction primarily and external medicine washing in cooperation for one year. In the early therapy stage, multiple types of physical therapy such as limb traction, acupuncture and infrared pain treatment were specifically selectively given in accordance with the status of patients. In addition, all patients were arranged with routine slowwalking exercises. Detailed information and follow-up conditions of each patient are presented in Table 1 (patients are denoted by PI-PVI respectively). The CT scan was applied at the initial presentation and follow-ups were performed at every 6-8 months for each patient which is the clinically routine follow-up period. We chose the clinical results at a uniform time point (one-year follow-up) of the patients as outcomes, which have been recorded. The bone collapse was found in two of the patients; thus, the six patients are divided into the non-collapse group (PI-IV) and the collapse group (PV and $\mathrm{PVI}$ ). Finite element analyses are performed on the pre-treatment (initial presentation) and post-treatment (6-8 months follow-up) models and are compared to the one-year clinical outcomes.

Table 1

Information and follow-up outcome of the patients

\begin{tabular}{|llllll|}
\hline Case ID & Age & Sex & Weight [kg] & Necrosis position & Outcome of 1-year follow-up \\
\hline PI & 55 & M & 65 & Right & non-collapse \\
\hline PII & 36 & M & 75 & Right & non-collapse \\
\hline PIII & 41 & M & 91 & Right & non-collapse \\
\hline PIV & 50 & F & 70 & Right & non-collapse \\
PV & 37 & M & 73 & Right & collapse \\
\hline PVI & 44 & M & 61 & Right & collapse \\
\hline
\end{tabular}

Models of proximal femurs were reconstructed in MIMICS 19.0 (Materialise, Belgium). The inner area (Fig. 1, red arrow) along edge of sclerotic band (Fig. 1, white arrow) allowing light to pass through but not transparent, named as low-density necrotic volume (LDNV) and the overall configuration of proximal femur were segmented from CT images as two 
independent parts by experienced doctors to ensure the repeatability. To guarantee the accuracy of the bone boundary, the 3D models were mapped back to CT images to check the outline fitness. Once the LDNV was reconstructed, the complementary healthy proximal femur was then subtracted by the overall proximal femur model by employing the Boolean algorithm. Therefore, two segments - the LDNV and the healthy proximal femur (viable portion)- were the two key finite element models of each case. Figure 2 displays the proximal femur models of the six patients pre- and posttreatment respectively (the LDNV is marked in red). These 12 models were discretized in Mechanical APDL (ANSYS 14.0, ANSYS Inc, USA) with 10-node tetrahedral elements. Grid resolution of both LDNV and the healthy proximal femur complementary to LDNV has been tested in the grid sensitivity analysis [49].

\subsection{Mechanical property assignment based on Hounsfield unit}

The bone density is linearly correlated to the Hounsfield unit (HU) represented by various function in specific locations and Young's modulus is determined by the local density [37, 40,43]. Meanwhile, one recent experimental research investigated the relationship between the density and elastic modulus in necrotic human femoral heads which proved that the necrotic region also follows an explicitly linear relationship similar to viable zones [56]. Moreover, the elastic modulus of necrotic zones determined from CT density values has been used in finite element modelling [48]. The coronal cross-sectional distribution of density and Young's Modulus in PV was shown in Fig. 3a as a representative illustration. The Young's modulus of the LDNV varies from 0.46 to $2.61 \mathrm{GPa}$; and that for the healthy proximal femur varies between 0.31 to $5.62 \mathrm{GPa}$.

\subsection{Numerical model and boundary conditions}

The force transformed on proximal part of the femur mainly from the hip joint and the connected ligament while the forces generated by other tissues could be neglected [30]. Here, to mimic slow-walking status, the peak loading is assigned as the resultant compressive force according to the weight of each patient $[1,33,46]$. The contact region between the femoral head and the hip is approximately $40^{\circ}$ of the central angle in the anterior-posterior plane and $80^{\circ}$ of the central angle in the medial-lateral plane (Fig. 3b) [1]. The zero-displacement boundary condition was applied at the distal end of the proximal femur model. Linear static numerical analysis of each model was carried out by a Finite Element solver, WORKBENCH 14.0 (ANSYS 14.0 Inc, Canonsburg, USA).

\section{Results}

In the current study, six patients with ARCO stage-II ONFH were studied from both morphological and mechanical perspective. Non-collapse cases reported positive situations, i.e. clinical symptoms and the range of motion of the hip joint activity, of the femoral head after one year; however, two presented bone collapse.

\subsection{Morphologic change}

Reconstructed models shows that the size of the femoral head varies among patients. Considering this individual difference, we defined another parameter named the volume ratio, i.e. the ratio between the LDNV and the entire femoral head volume. The volume of the femoral head $\left(\mathrm{V}_{\mathrm{FH}}\right)$ was calculated by cropping the model through a plane that is perpendicular to the anterior-posterior plane, perpendicular to the proximal and distal c-curves of the femoral neck, and crosses the trough of the distal femoral neck c-curve. The averaged value of $\mathrm{V}_{\mathrm{FH}}$ of pre- and post-treatment was applied as the $\mathrm{V}_{\mathrm{FH}}$ for each patient and the volume of necrosis $\left(\mathrm{V}_{\mathrm{N}}\right)$ is compared to $\mathrm{V}_{\mathrm{FH}}$ in order to analyze the morphological variation after medical treatment. The results are listed in Table 2. Reduction of the LDNV volume ratio is found in PI, PIV, PV and PVl; however, the ratio increase is found in PII and PIII. Comparing to follow-up results shown in Table 1 (bone collapse is found in PV and PVI), the LDNV with non-collapse consequence indeed increased in PII and PIII while that reduced in PV and PVI. This result indicates the isolated morphological variation may not a reliable index to predict the collapse of the ONFH and volume-based evaluation of the medical treatment may not be effective. 
Table 2

Morphological and mechanical variation after medical treatment

\begin{tabular}{|c|c|c|c|c|c|c|c|c|c|}
\hline Case & Status & $\begin{array}{l}V_{F H} \\
{\left[\times 10^{4} \mathrm{~mm}^{3}\right]}\end{array}$ & $\begin{array}{l}V_{N} \\
{\left[\times 10^{4} \mathrm{~mm}^{3}\right]}\end{array}$ & $\begin{array}{l}\text { Volume } \\
\text { Ratio } \\
\text { [\%] }\end{array}$ & $\begin{array}{l}\text { Volume } \\
\text { ratio } \\
\text { variation } \\
\text { [\%] }\end{array}$ & $\begin{array}{l}\text { Averaged } \\
\text { Equivalent } \\
\text { Stress } \\
\text { [MPa] }\end{array}$ & $\begin{array}{l}\text { Averaged } \\
\text { Equivalent } \\
\text { Stress } \\
\text { Variation } \\
\text { [\%] }\end{array}$ & $\begin{array}{l}\text { Relative } \\
\text { Stress } \\
\text { Index } \\
\text { ()) } \\
{\left[\mathrm{x}^{-}\right.} \\
{\left[\mathrm{x}^{-}\right.} \\
\left.{ }^{3} \mathrm{~Pa}^{-1}\right]\end{array}$ & $\begin{array}{l}\text { Relative } \\
\text { Stress } \\
\text { Index } \\
\text { Variation } \\
\text { [\%] }\end{array}$ \\
\hline \multirow[t]{2}{*}{$\mathrm{PI}$} & Pre & \multirow[t]{2}{*}{5.4844} & 1.2750 & 23.25 & \multirow[t]{2}{*}{-3.15} & 0.7370 & \multirow[t]{2}{*}{9.48} & 5.87 & \multirow[t]{2}{*}{-6.13} \\
\hline & Post & & 1.1024 & 20.10 & & 0.8069 & & 5.51 & \\
\hline \multirow[t]{2}{*}{ PII } & Pre & \multirow[t]{2}{*}{6.5231} & 0.9236 & 14.16 & \multirow[t]{2}{*}{3.56} & 0.7905 & \multirow[t]{2}{*}{-3.98} & 5.64 & \multirow[t]{2}{*}{-0.71} \\
\hline & Post & & 1.1560 & 17.72 & & 0.7590 & & 5.60 & \\
\hline \multirow[t]{2}{*}{ PIII } & Pre & \multirow[t]{2}{*}{5.7722} & 0.2485 & 4.31 & \multirow[t]{2}{*}{1.97} & 0.9584 & \multirow[t]{2}{*}{4.57} & 6.03 & \multirow[t]{2}{*}{-2.49} \\
\hline & Post & & 0.3623 & 6.28 & & 1.0022 & & 5.88 & \\
\hline \multirow[t]{2}{*}{ PIV } & Pre & \multirow[t]{2}{*}{4.8993} & 0.6158 & 12.57 & \multirow[t]{2}{*}{-1.01} & 0.8487 & \multirow[t]{2}{*}{6.42} & 6.40 & \multirow[t]{2}{*}{0.47} \\
\hline & Post & & 0.5662 & 11.56 & & 0.9032 & & 6.43 & \\
\hline \multirow[t]{2}{*}{ PV } & Pre & \multirow[t]{2}{*}{6.0108} & 1.9734 & 32.83 & \multirow[t]{2}{*}{-7.56} & 1.0616 & \multirow[t]{2}{*}{2.94} & 5.93 & \multirow[t]{2}{*}{7.42} \\
\hline & Post & & 1.5187 & 25.27 & & 1.0928 & & 6.37 & \\
\hline \multirow[t]{2}{*}{ PVI } & Pre & \multirow[t]{2}{*}{5.1976} & 2.6758 & 51.48 & \multirow[t]{2}{*}{-7.33} & 1.2851 & \multirow[t]{2}{*}{2.01} & 6.08 & \multirow[t]{2}{*}{1.15} \\
\hline & Post & & 2.2946 & 44.15 & & 1.3109 & & 6.15 & \\
\hline
\end{tabular}

\subsection{Loading distribution}

The bone strength could be directly represented by the mechanical distribution on bone structure from finite element simulations. Figure $4 a-b$ displays the equivalent stress distribution on a slice of the bone model along the coronal plane for PII (uncollapsed during follow-up) and PV (collapsed during follow-up) as representatives. While Fig. 4c shows the equivalent stress distribution on the coronal plane of a healthy femur (the healthy femur is modeled based on the CT images of the normal side of a patient). In healthy cases, the major stress path of the femoral head is consecutively transferred downwards. High stress on the slice occurs along the direction of the principal trabecular system, on the superior and posterior surface of the femur neck and the peripheral region of the femur shaft. However, the stress transfer path of the cases with ONFH is notably distinct. As shown in Fig. 4a, high stress is often occurred around the principal trabecular system and the central region of the femoral neck (arrow indicated), indicating potential risk at the Ward triangle due to this abnormal mechanical load. After medical treatment (Fig. 4b), high stress in the central region of the femoral head is reduced in PI-IV; however, the stress variation of this region in PV and PVI is not obvious. Comparing to the stress distribution in the healthy cases, the mechanical distribution for the load-bearing in PI-IV is better improved. This is truly consistent with the one-year follow-up results.

More specifically, distributions of equivalent stress of the necrotic region of the patients were plotted in Fig. 5. The upper panel (Fig. 5a) displays the equivalent stress distribution of the LDNV before treatment while the lower panel (Fig. 5b) shows the results after treatments. In all of the cases, the highest stress of the LDNV is found in the posterior portion of the interface between the necrosis and the healthy proximal femur. This is consistent with previous studies, which found that the collapse of the necrotic femoral head is caused by a series of fatigue fracture and the fracture in the necrotic region mostly appears in the posterior region of the underlying necrotic-viable interface [54]. After medical treatment, the highest stress in this region is increased in PI, PIII, PIV and PVI; whereas, it reduces in PII and PV. On the other hand, the 
averaged equivalent stress for each model is shown in Table 2. The averaged equivalent stress increases in most of the post-treatment cases; however, in PII, this value reduces after treatment. Thus, similar to the results of volume ratio change, both the variation of the highest and averaged equivalent stress in necrosis between pre- and post-treatment models do not show direct correlations with the follow-up results.

Directly, the finite element analysis could provide the equivalent stress, which might cannot accurately reveal the detailed mechanical behavior individually. Hence, we defined an stress index (SI), i.e. the ratio between equivalent stress $\left(\sigma_{\mathrm{e}}\right)$ and compressive strength $\left(\sigma_{\mathrm{c}}\right)$, where the later is the mean stress index related to the local density of the material. Literature regarding the density-compressive strength relationship of the necrosis are lacking. Since the loose structure of the necrotic region is relatively similar to the cancellous bone, the density-compressive strength relationship of the cancellous bone in the axial loading direction is applied, which can by described by Equ.1 [52], where $\sigma_{\mathrm{c}}(\mathrm{MPa})$ denotes compressive strength, $\rho\left(\mathrm{g} / \mathrm{cm}^{3}\right)$ denotes bone density. The mean equivalent stress $(s)$ and the mean stress index $(I)$ of the necrosis were defined in Equ.2, where $V$ denotes the volume.

$$
\begin{gathered}
\sigma_{c}=40.8 \rho^{189} \\
s=\frac{\int_{V} \sigma_{\varepsilon} d V}{\int_{V} d V}, \quad i=\frac{\int_{V}\left(\sigma_{\varepsilon} / \sigma_{c}\right) d V}{\int_{V} d V}
\end{gathered}
$$

We defined the ratio of $i$ and $s$, denoted as relative stress index $\left(R S I=i / s, \mathrm{~Pa}^{-1}\right)$, as a parameter to evaluate the effectiveness of medical treatment. The results are shown in Table 2. For the post-treatment cases, this ratio (RSI) of PIPIII is decreased; the ratio of PIV is slightly increased; while the ratio in PV and PVI is increased. Based on the definition of $R S I$, a lower value of $R S /$ indicates stronger mechanical loading capability of the bone. Thus, this reduction of $R S /$ value reveals the improvement of bone strength resulting from the positive affect of the conservative medical treatment. In fact, according to the follow-up results, PI-III received positive feedback and the bones are uncollapsed. Collapse is not found in PIV as well, although the $R S I$ of it is slightly increased by $0.47 \%$. Specifically, the LDNV locates at the anteromedial site on femoral head outside the loading region and thus the loading transmission status to the necrotic volume in PIV might be different from other cases.

\section{Discussion}

The credible evaluation of the effectiveness is of significant importance for the conservative medical treatment of ONFH before collapse. Clinical assessment is currently still relies on radiation scans and symptoms of patients, which includes pain feedback and physical limitations. Kerboul et al. estimated the extent of necrosis radiographically in the early-stages using the sum of the arc of the area of the femoral head involved on anteroposterior and lateral radiographs. They pointed out that clinical outcomes were more favourable if this value was $<200^{\circ}$ [25]. Yong-Chan Ha et al. modified Kerboul method using MRI and proposed necrotic index to evaluate the progression of ONFH and pointed that if the index was > $40 \%$, clinical outcomes were worse [15]. The medical images only provide morphologic information while the feedbacks from the patients is highly subjective.

The morphologic change of LDNV is one of the most important indices of the progression of ONFH [22]. However, the quantitative volume of LDNV can hardly be directly assessed by medical scans due to the highly individualized geometric feature of the necrotic region. Moreover, the area and volume ratio of the LDNV are usually calculated to estimate the extent of necrosis $[42,36,17]$. In this study, we firstly compared the morphological change of the LDNV pre-and posttreatment to investigate the effectiveness of the medical treatment. In the morphological comparison, shown in Table 2, the volume ratio of the LDNV decreases in four of the cases (PI, PIV, PV and PVI) in the follow-up models. However, the bone collapse was found in PV and PVI in the one-year follow-up, implicating that the morphological change alone may 
not sufficient to effectively evaluate the efficacy of the medical treatment and mechanical analysis needed to be incorporated. This result implies that even positive morphologic variations are found (LDNV decreases), the necrotic femoral head may still suffer from collapse risk.

Mechanical evaluations of the bone may assist in better estimation of the effectiveness of the medical treatment. Previously, various biomechanical studies have proposed that biomechanical properties related to treatment results can be provided by finite element analysis[27, 12,11$]$. In a large number of relevant studies, necrotic bone tissues were segmented into three regions: necrotic core, cancellous bone and cortical bone region, according to the CT datasets, and the materials for these three bone structures are treated as homogeneous material $[58,27,50,47,3]$. Since different levels of stiffness have been assigned in the three components of the bones, the simulation results would be highly dependent on the image segmentation. Thus, the disadvantage of this method is underestimating the load-bearing capability of the overall bone, e.g., the missing of the principal compressive trabecular bone whose major function is loading the principal compressive stress. The morphological characteristics and region of the principal compressive group are estimated by CT images (Fig. 4c).

In the current study, the HU-based Young's modulus was assigned to each discretized model which is able to satisfy the anisotropic feature of bone material, especially for the cancellous bone. The equivalent stress transfer path in the femoral head of the healthy case I is displayed in Fig. 3a, which demonstrates that the region of the equivalent stress transfer path is consistent with the cross-section of CT image. Hence, the finite element results of the present mechanical study appear to reflect the physical phenomenon of the femur.

Loading boundary conditions were imposed based on patient-specific weight to take account of the individual condition. As shown in Fig. 4, the difference of the equivalent stress distribution on the coronal plane of the proximal femur between the healthy and ONFH cases is mainly presented in the region of the femoral neck. Ward's triangle is a space localized at the femoral neck formed by the intersection of the compressive trabeculae groups and the tensile trabeculae groups defining a neutral axis where tensile and compressive forces balance each other. Ward's triangle rarely bears stress in the normal condition [32]. In healthy cases, in the present FE study, high stress is transferred along with the principal compressive group. Some previous results displayed the similar stress patterns with the finite element results in the present study $[58,21,31,2,20]$. While as arrows indicated, in the ONFH cases, the high equivalent stress occurs at the central region of the femoral neck close to Ward triangle, where the distribution in ONFH may induce a potential risk of the bone collapse. After treatment, the non-collapse group (PI-IV) presents an obvious reduction of the stress near the Ward triangle indicating the trend of normalization after medical treatment. However, there is no noticeable change showed in the collapse group (PV and PVI), indicating the stress distribution in the area of Ward triangle may be potential to reveal the efficacy of medical treatment from the mechanical point of view. Furthermore, the mechanical parameters of the necrosis are investigated. High stress occurs in the posterior region of the necrosis that is consistent with the actual fracture region of the ONFH in clinical reports. However, the variation of the LDNV stress pre- and post- medical treatment does not show correspondence to the clinical follow-up results. A previous study investigated the stress and strength distribution on various sizes of necrotic areas by finite element models to predict the fate of the femoral head for earlystage osteonecrosis [54]. An analysis including 28 osteonecrotic femoral head specimens pointed out that the major fracture site appeared at the deep necrotic region of the interface between the necrosis and the healthy proximal femur. The ratio of the equivalent stress and yield strength of the necrosis was calculated. The results exhibited that the site of fracture coincided with the region of the ratio greater than the physiological level ( 0.10 or less they pointed) in the finite element model study. Thus, the mechanical properties of the necrosis can be represented by the stress and strength of the region. According to published studies, the compressive strength of cancellous femoral bone in the axial load direction is dependent on local density $[52,28,29]$. Thus, the compressive strength of the LDNV is calculated according to the functional relations. In the current study, based on the equivalent stress and compressive strength, averaged equivalent stress, as well as the averaged stress index in the LDNV, were calculated and compared. By calculating the ratio (RSI)

Page $7 / 16$ 
between the averaged stress index and equivalent stress, results show that the ratio in most cases of the non-collapse group reduces after treatment; while it increases in the collapse group. In fact, a smaller value of the $R S /$ indicates higher compressive strength, which is directly related to the density of the material; on the other hand, larger $R S /$ indicates smaller compressive strength and thus may be related to the risk of bone collapse.

Morphological results confirm the lack of capability of isolated morphological analysis in evaluating the effectiveness of the medical treatment on ONFH. The finite element analysis seems to be a good non-invasive tool to model the mechanical properties of the femoral and to provide a quantitative evaluation of the load distribution over the proximal femur. Therefore, it has the potential to predict bone collapse risk and to estimate the treatment effects. The currently study preliminarily proposed the relative stress index $(R S I)$ and the distribution of equivalent stress at the Ward triangle as the mechanical parameters that are possible to evaluate medical treatment results.

It should be noted that this study is based on limited patient cases which is due to the rareness of such cases who merely treated by pharmacotherapy without any intervention. To achieve the predictability of such parameter, the proposed $R S I$, it needs to be validated by larger patient datasets. Further confirmations of the predictive ability of these parameters will be conducted in the near future.

\section{Conclusions}

We enrolled six patients with ONFH pre- and post-medical treatment and the mechanical behavior was compared. Both the morphologic and mechanical variation has been calculated and discussed. The relative stress index (RSI), defined as the ratio between the mean stress index of volume and the mean equivalent stress of volume, is proposed to be potential to evaluate the medical treatment effect. The variation of equivalent stress at the Ward triangle is also found related to the medical treatment outcomes. This study indicates morphological analysis alone cannot estimate the medical treatment results effectively; mechanical analysis is needed, which may assist in improving the safety of the treatment and in early medical decision-making on surgery or intervention.

\section{Abbreviations}

\section{ONFH}

Osteonecrosis of the femoral head

\section{ARCO}

Association Research Circulation Osseous

THR

The total hip replacement

\section{LDNV}

Low-density necrotic volume

HU

Hounsfield unit

$\mathrm{V}_{\mathrm{FH}}$

The volume of the femoral head

$\mathrm{V}_{\mathrm{N}}$

The volume of necrosis

SI

Stress index

RSI

The relative stress index 


\section{Declarations}

\section{Ethics approval and consent to participate}

Approval of the local ethic committee by China Academy of Chinese Medical Science (WJEC-KT-2016-008-P002) and informed consent from the examined patients were obtained prior to the simulation study.

\section{Consent for publication}

Approval of the local ethic committee by China Academy of Chinese Medical Science (WJEC-KT-2016-008-P002) and informed consent to publish the result in the study obtained from participants.

\section{Availability of data and materials}

The datasets generated and analyzed during the current study are collected from hospital, which are privacy data of patients. Hence, it only can be obtained from corresponding author.

\section{Competing interests}

The authors declare that they have no competing interests.

\section{Funding}

This study is supported by the National Natural Science Foundation of China (Nos. 81373801 and 81471752 ), the Natural Science Foundation of Beijing (No. 7142170) and the National Science \& Technology Pillar Program (2015BAI04B03).

\section{Authors' contributions}

Yuqian Mei carried out the finite element computations, data analysis and drafted the manuscript. Qi Tang and Songhao Chen carried out the image segmentation and 3D model reconstruction and participated in mechanical analysis of the models. Prof Duanduan Chen designed this study, directed the mechanical simulations and helped to draft the manuscript.

\section{Acknowledgements}

Thanks for Wang Jing hospital providing data. This study is supported by the National Key R\&D Program of China (No. 2018AAA0102600), the National Natural Science Foundation of China (Nos. 81970404 and No.81973888), Beijing Nova Program (No. Z181100006218008), Beijing Municipal Science \& Technology Commission (No. Z191100010618004), and Beijing Municipal Natural Science Foundation of China (No. 7182186).

\section{Author information}

\section{Affiliations}

1. School of Life Science, Beijing Institute of Technology, China

Yuqian Mei, Qi Tang, Songhao Chen, \&Duanduan Chen

2. Key Laboratory of Convergence Medical Engineering System and Healthcare Technology, The Ministry of Industry and Information Technology, Beijing Institute of Technology, China Anna Yuqian Mei \& Duanduan Chen 


\section{References}

1. Bergmann G, Deuretzbacher G, Heller M, Graichen F, Rohlmann A, Strauss J, Duda G (2001) Hip contact forces and gait patterns from routine activities. Journal of biomechanics 34:859-871. http://dx.doi.org/10.1016/S00219290(01)00040-9

2. Boyle C, Kim IY (2011) Three-dimensional micro-level computational study of Wolff's law via trabecular bone remodeling in the human proximal femur using design space topology optimization. Journal of biomechanics 44:935-942. http://dx.doi.org/10.1016/j.jbiomech.2010.11.029

3. Brown T, Hild G (1983) Pre-collapse stress redistributions in femoral head osteonecrosis-a three-dimensional finite element analysis. J Biomech Eng. http://dx.doi.org/10.1115/1.3138402

4. Brown TD, Pedersen DR, Baker KJ, Brand RA (1993) Mechanical consequences of core drilling and bone-grafting on osteonecrosis of the femoral head. The Journal of bone joint surgery American volume 75:1358-1367. http://dx.doi.org/10.2106/00004623-199309000-00011

5. Bryan R, Nair PB, Taylor M (2012) Influence of femur size and morphology on load transfer in the resurfaced femoral head: A large scale, multi-subject finite element study. Journal of biomechanics 45:1952-1958. http://dx.doi.org/10.1016/j.jbiomech.2012.05.015

6. Camp JF, Colwell JC (1986) Core decompression of the femoral head for osteonecrosis. The Journal of bone joint surgery American volume 68:1313-1319. http://dx.doi.org/10.2106/00004623-198668090-00002

7. Chandler HP, Reineck F, Wixson R, McCarthy J (1981) Total hip replacement in patients younger than thirty years old. A five-year follow-up study. JBJS 63:1462-1434. http://dx.doi.org/10.2106/00004623-198163090-00008

8. Chen W-P, Tai C-L, Shih C-H, Hsieh P-H, Leou M-C, Lee MS (2004) Selection of fixation devices in proximal femur rotational osteotomy: clinical complications and finite element analysis. Clinical Biomechanics 19:255-262. http://dx.doi.org/10.1016/j.clinbiomech.2003.12.003

9. Cherian SF, Laorr A, Saleh KJ, Kuskowski MA, Bailey RF, Cheng EY (2003) Quantifying the extent of femoral head involvement in osteonecrosis. JBJS 85:309-315. http://dx.doi.org/10.2106/00004623-200302000-00019

10. Cornell C, Salvati E, Pellicci P (1985) Long-term follow-up of total hip replacement in patients with osteonecrosis. The Orthopedic clinics of North America 16:757-769

11. Daniel M, Herman S, Dolinar D, Iglic A, Sochor M, Kralj-Iglic V (2006) Contact stress in hips with osteonecrosis of the femoral head. Clinical Orthopaedics Related Research® 447:92-99. http://dx.doi.org/10.1097/01.blo.0000203472.88926.c8

12. Floerkemeier T, Lutz A, Nackenhorst U, Thorey F, Waizy H, Windhagen H, von Lewinski G (2011) Core decompression and osteonecrosis intervention rod in osteonecrosis of the femoral head: clinical outcome and finite element analysis. International orthopaedics 35:1461-1466. http://dx.doi.org/10.1007/s00264-010-1138-x

13. Floerkemeier T, Thorey F, Daentzer D, Lerch M, Klages P, Windhagen H, von Lewinski G (2011) Clinical and radiological outcome of the treatment of osteonecrosis of the femoral head using the osteonecrosis intervention implant. International orthopaedics 35:489-495. http://dx.doi.org/10.1007/s00264-009-0940-9

14. Garcia J, Doblaré M, Cegonino J (2002) Bone remodelling simulation: a tool for implant design. Computational materials science 25:100-114. http://dx.doi.org/10.1016/S0927-0256(02)00254-9

15. Ha Y-C, Lee Y-K, Koo K-H (2014) Modified Kerboul Classification System of Necrotic Extent Using MRI. In: Osteonecrosis. Springer, pp 211-213

16. Herndon JH, Aufranc OEJCO (1972) Avascular necrosis of the femoral head in the adult: a review of its incidence in a variety of conditions. J Clinical Orthopaedics Related Research® 86:43-62. doi:10.1097/00003086-197207000- 
00008. http://dx.doi.org/10.1097/00003086-197207000-00008

17. Hernigou P, Lambotte J (2001) Volumetric analysis of osteonecrosis of the femur: anatomical correlation using MRI. The Journal of bone joint surgery British volume 83:672-675. http://dx.doi.org/10.1302/0301-620X.83B5.0830672

18. Hu L, Huang Z, Wei H, Wang W, Ren A, Xu Y (2015) Osteonecrosis of the femoral head: using CT, MRI and gross specimen to characterize the location, shape and size of the lesion. The British journal of radiology 88:20140508

19. Hungerford DS (2007) Treatment of osteonecrosis of the femoral head: everything's new. The Journal of arthroplasty 22:91-94

20. Jang IG, Kim IY (2008) Computational study of Wolff's law with trabecular architecture in the human proximal femur using topology optimization. Journal of Biomechanics 41:2353-2361.

http://dx.doi.org/10.1016/j.jbiomech.2008.05.037

21. Jang IG, Kim IY (2009) Computational simulation of trabecular adaptation progress in human proximal femur during growth. Journal of Biomechanics 42:573-580. http://dx.doi.org/10.1016/j.jbiomech.2008.12.009

22. Jawad MU, Haleem AA, Scully SP (2012) In brief: Ficat classification: avascular necrosis of the femoral head. Springer, Clinical Orthopaedics and Related Research®. http://dx.doi.org/10.1007/s11999-012-2416-2

23. Jiang Y, Liu D, Kong X, Xu Y, Chen W, Lin NJJoTCM (2014) Huogu I formula prevents steroid-induced osteonecrosis in rats by down-regulating PPARY expression and activating Wnt/LRP5/ $\beta$-catenin signaling. Journal of Traditional Chinese Medicine 34:342-350. http://dx.doi.org/10.1016/S0254-6272(14)60100-X

24. Kato H, Nakamura T, Nishiguchi S, Matsusue Y, Kobayashi M, Miyazaki T, Kim HM, Kokubo T (2000) Bonding of alkaliand heat-treated tantalum implants to bone. Journal of Biomedical Materials Research 53:28-35.

http://dx.doi.org/10.1002/(SICI)1097-4636(2000)53:1\%3C28::AID-JBM4\%3E3.0.CO;2-F

25. Kerboul M, Thomine J, Postel M, d'Aubigne RM (1974) The conservative surgical treatment of idiopathic aseptic necrosis of the femoral head. The Journal of bone joint surgery British volume 56:291-296. doi:doi: 10.1302/0301620x.56b2.291. http://dx.doi.org/10.1302/0301-620X.56B2.291

26. Kim Y-H, Kim J-S, Park J-W, Joo J-H (2011) Contemporary total hip arthroplasty with and without cement in patients with osteonecrosis of the femoral head: a concise follow-up, at an average of seventeen years, of a previous report. JBJS 93:1806-1810. http://dx.doi.org/10.2106/JBJS.J.01312

27. Lee MS, Tai C-L, Senan V, Shih C-H, Lo S-W, Chen W-P (2006) The effect of necrotic lesion size and rotational degree on the stress reduction in transtrochanteric rotational osteotomy for femoral head osteonecrosis-a three-dimensional finite-element simulation. Clinical Biomechanics 21:969-976

28. Lotz JC, Gerhart TN, Hayes WC (1990) Mechanical properties of trabecular bone from the proximal femur: a quantitative CT study. Journal of computer assisted tomography 14:107-114. http://dx.doi.org/10.1097/00004728199001000-00020

29. Lotz JC, Gerhart TN, Hayes WC (1991) Mechanical properties of metaphyseal bone in the proximal femur. Journal of biomechanics 24:317-329. http://dx.doi.org/10.1016/0021-9290(91)90350-V

30. Lutz A, Nackenhorst U, von Lewinski G, Windhagen H, Floerkemeier T (2011) Numerical studies on alternative therapies for femoral head necrosis. Biomechanics modeling in mechanobiology 10:627-640.

http://dx.doi.org/10.1007/s10237-010-0261-3

31. Ma X, Li H, Ma J, CHEN Y, ZHU S-w, MA B-y, YANG Y, MA T, WANG J, WANG Z-b (2012) Biomechanical properties of principle compressive trabecular bone in proximal femur. Biome Eng Clin Med 2:118-122

32. Machado M, Fernandes P, Cardadeiro G, Baptista F (2013) Femoral neck bone adaptation to weight-bearing physical activity by computational analysis. Journal of biomechanics 46:2179-2185.

http://dx.doi.org/10.1016/j.jbiomech.2013.06.031

Page $11 / 16$ 
33. Machado M, Fernandes P, Zymbal V, Baptista F (2014) Human proximal femur bone adaptation to variations in hip geometry. Bone 67:193-199. http://dx.doi.org/10.1016/j.bone.2014.07.001

34. Mann K, Bartel D, Wright T, Burstein A (1995) Coulomb frictional interfaces in modeling cemented total hip replacements: a more realistic model. Journal of Biomechanics 28:1067-1078 http://dx.doi.org/10.1016/00219290(94)00158-Z.

35. Mont MA, Ragland PS, Etienne G (2004) Core decompression of the femoral head for osteonecrosis using percutaneous multiple small-diameter drilling. Clinical Orthopaedics Related Research 429:131-138. http://dx.doi.org/10.1097/01.blo.0000150128.57777.8e

36. Nishii T, Sugano N, Ohzono K, Sakai T, Sato Y, Yoshikawa H (2002) Significance of lesion size and location in the prediction of collapse of osteonecrosis of the femoral head: a new three-dimensional quantification using magnetic resonance imaging. Journal of orthopaedic research 20:130-136. http://dx.doi.org/10.1016/S0736-0266(01)00063-8

37. Peng L, Bai J, Zeng X, Zhou Y (2006) Comparison of isotropic and orthotropic material property assignments on femoral finite element models under two loading conditions. Medical engineering physics 28:227-233. http://dx.doi.org/10.1016/j.medengphy.2005.06.003

38. Pérez M, Vendittoli P-A, Lavigne M, Nuño N (2014) Bone remodeling in the resurfaced femoral head: Effect of cement mantle thickness and interface characteristics. Medical engineering physics 36:185-195. http://dx.doi.org/10.1016/j.medengphy.2013.10.013

39. Polkowski GG, Callaghan JJ, Mont MA, Clohisy JC (2012) Total hip arthroplasty in the very young patient. JAAOSJournal of the American Academy of Orthopaedic Surgeons 20:487-497. http://dx.doi.org/10.5435/JAAOS-20-08487

40. Rho J-Y, Hobatho M, Ashman R (1995) Relations of mechanical properties to density and CT numbers in human bone. Medical engineering physics 17:347-355

41. Stauffer R (1982) Ten-year follow-up study of total hip replacement. The Journal of bone joint surgery American volume 64:983-990. http://dx.doi.org/10.2106/00004623-198264070-00003

42. Sugano N, Takaoka K, Ohzono K, Matsui M, Masuhara K, Ono K (1994) Prognostication of nontraumatic avascular necrosis of the femoral head. Significance of location and size of the necrotic lesion. Clinical orthopaedics related research:155-164

43. Taylor W, Roland E, Ploeg H, Hertig D, Klabunde R, Warner M, Hobatho M, Rakotomanana L, Clift S (2002) Determination of orthotropic bone elastic constants using FEA and modal analysis. Journal of biomechanics 35:767-773. http://dx.doi.org/10.1016/S0021-9290(02)00022-2

44. Tran T, Warwas S, Haversath M, Classen T, Hohn H, Jäger M, Kowalczyk W, Landgraeber S (2014) Experimental and computational studies on the femoral fracture risk for advanced core decompression. Clinical Biomechanics 29:412417. http://dx.doi.org/10.1016/j.clinbiomech.2014.02.001

45. Tripathy SK, Goyal T, Sen RK (2015) Management of femoral head osteonecrosis: Current concepts. Indian journal of orthopaedics 49:28. http://dx.doi.org/10.4103/0019-5413.143911

46. Tsuji T, Sugano N, Sakai T, Yoshikawa H (2003) Evaluation of femoral perfusion in a non-traumatic rabbit osteonecrosis model with T2*-weighted dynamic MRI. Journal of orthopaedic research 21:341-351. http://dx.doi.org/10.1016/S0736-0266(02)00144-4

47. Ueo T, Tsutsumi S, Yamamuro T, Okumura H, Shimizu A, Nakamura T (1985) Biomechanical aspects of the development of aseptic necrosis of the femoral head. Archives of orthopaedic traumatic surgery 104:145-149. http://dx.doi.org/10.1007/BF00454690

48. Utsunomiya T, Motomura G, Ikemura S, Kubo Y, Sonoda K, Hatanaka H, Baba S, Kawano K, Yamamoto T, Nakashima Y (2018) Effects of sclerotic changes on stress concentration in early-stage osteonecrosis: A patient-specific, 3D finite element analysis. Journal of Orthopaedic Research® 36:3169-3177. http://dx.doi.org/10.1002/jor.24124

Page $12 / 16$ 
49. Viceconti M, Olsen S, Nolte L-P, Burton K (2005) Extracting clinically relevant data from finite element simulations. Clinical Biomechanics 20:451-454. http://dx.doi.org/10.1016/j.clinbiomech.2005.01.010

50. Volokh K, Yoshida H, Leali A, Fetto J, Chao E (2005) Prediction of femoral head collapse in osteonecrosis. J Biomech Eng. http://dx.doi.org/10.1115/1.2187050

51. Wan F, Zhang Q, Wen P, Cheng L, Li P, Guo W (2018) Effect of glucocorticoids on miRNA expression spectrum of rat femoral head microcirculation endothelial cells. Gene 651:126-133

52. Wirtz DC, Schiffers N, Pandorf T, Radermacher K, Weichert D, Forst R (2000) Critical evaluation of known bone material properties to realize anisotropic FE-simulation of the proximal femur. Journal of biomechanics 33:13251330. http://dx.doi.org/10.1016/S0021-9290(00)00069-5

53. Wu W, He W, Wei Q-S, Chen Z-Q, Gao D-W, Chen P, Zhang Q-W, Fang B, Chen L-I, Li B-L (2018) Prognostic analysis of different morphology of the necrotic-viable interface in osteonecrosis of the femoral head. International orthopaedics 42:133-139. http://dx.doi.org/10.1007/s00264-017-3679-8

54. Yang J-W, Koo K-H, Lee M-C, Yang P, Noh MD, Kim S-Y, Kim K-I, Ha Y-C, Joun M-S (2002) Mechanics of femoral head osteonecrosis using three-dimensional finite element method. J Archives of orthopaedic trauma surgery 122:88-92. http://dx.doi.org/10.1007/s004020100324

55. Yu T, Xie L, Zhang Z, Ke X, Liu Y (2015) Prediction of osteonecrosis collapse of the femoral head based on the proportion of the proximal sclerotic rim. International orthopaedics 39:1045-1050. http://dx.doi.org/10.1007/s00264015-2679-9

56. Yue Y, Yang H, Li Y, Zhong H, Tang Q, Wang J, Wang R, He H, Chen W, Chen D (2019) Combining ultrasonic and computed tomography scanning to characterize mechanical properties of cancellous bone in necrotic human femoral heads. Medical engineering physics 66:12-17. http://dx.doi.org/10.1016/j.medengphy.2019.02.002

57. Zhao Dw, Hu Yc (2012) Chinese experts' consensus on the diagnosis and treatment of osteonecrosis of the femoral head in adults. Orthopaedic surgery 4:125-130. http://dx.doi.org/10.1111/j.1757-7861.2012.00192.x

58. Zhou G-Q, Pang Z-H, Chen Q-Q, He W, Chen Z-Q, Chen L-L, Li Z-Q (2014) Reconstruction of the biomechanical transfer path of femoral head necrosis: A subject-specific finite element investigation. Computers in biology medicine 52:96101

\section{Figures}
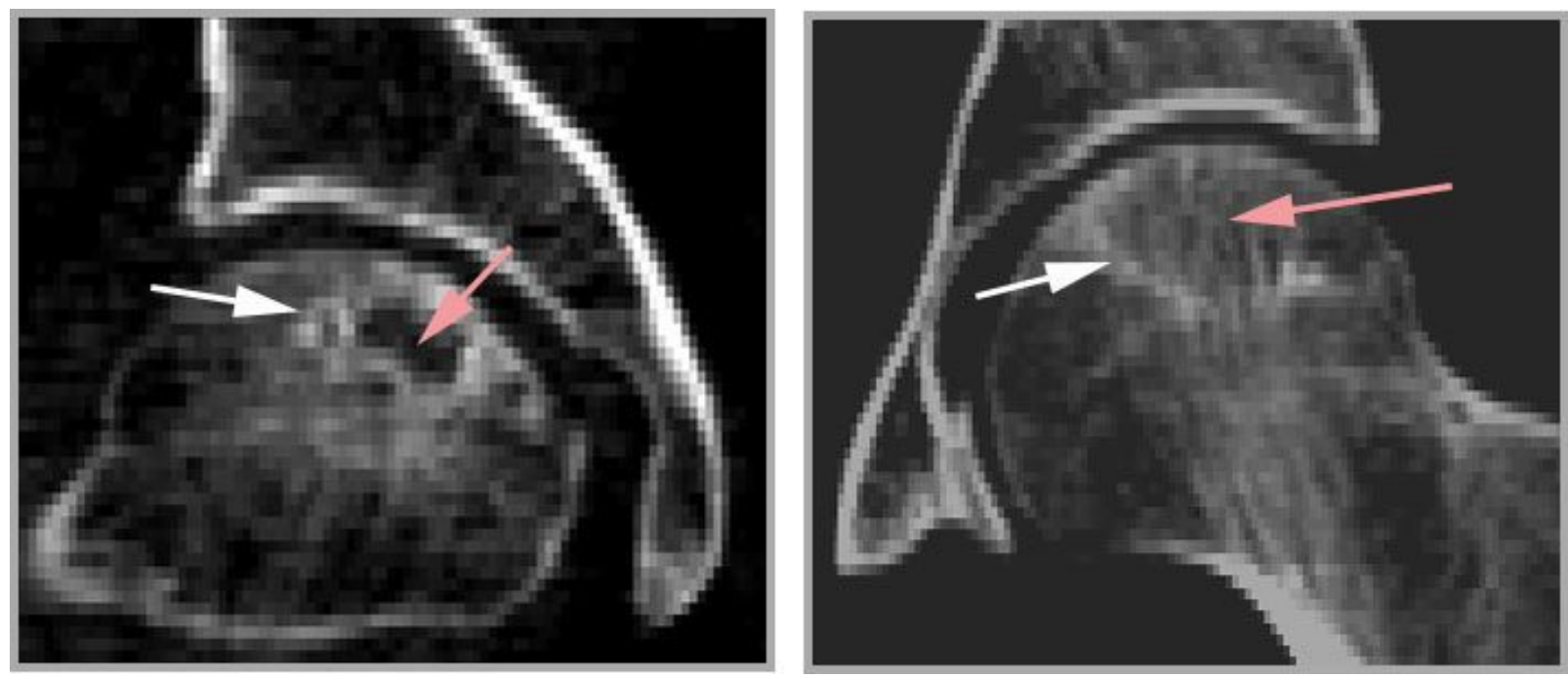

Figure 1 
Representative slides of coronal CT scan of ONFH indicating the low-density necrotic region (white arrow) inside the edge of sclerotic band (red arrow)

\section{(a) Pre-treatment}
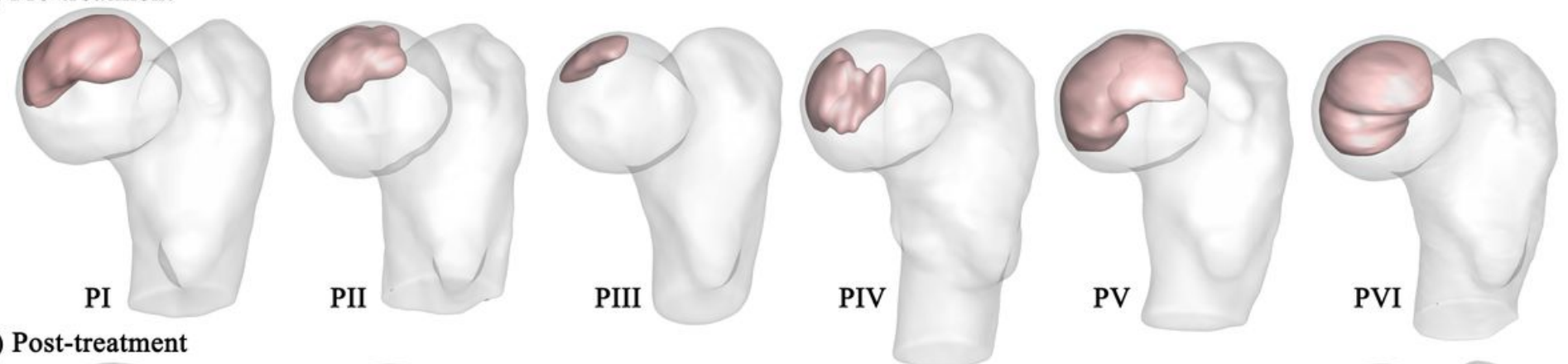

(b) Post-treatment
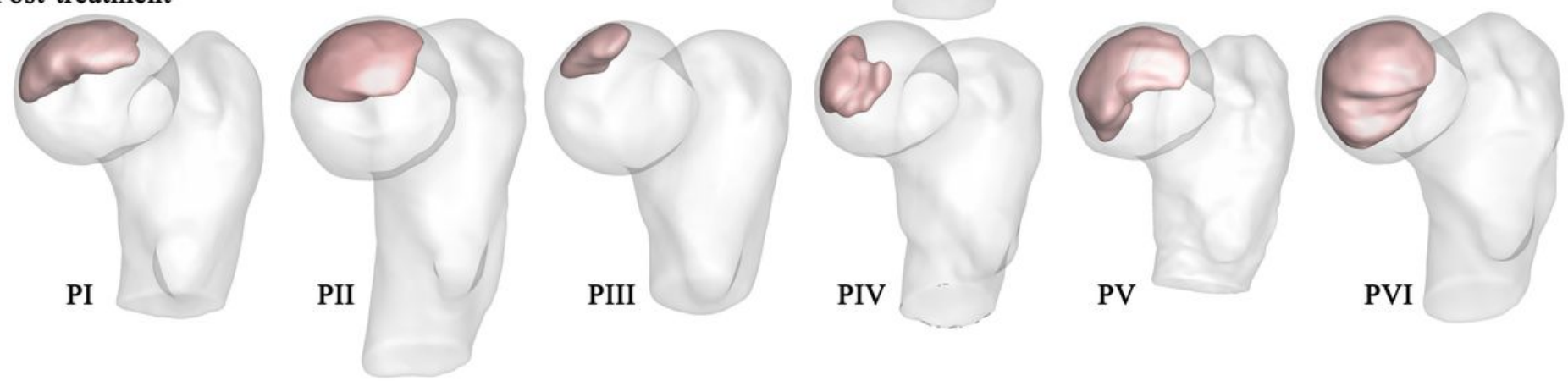

Figure 2

Patient-specific models of ONFH. (a) shows the pre-treatment models at initial presentation and (b) displays the posttreatment models at 6-8 months follow-up

(a)

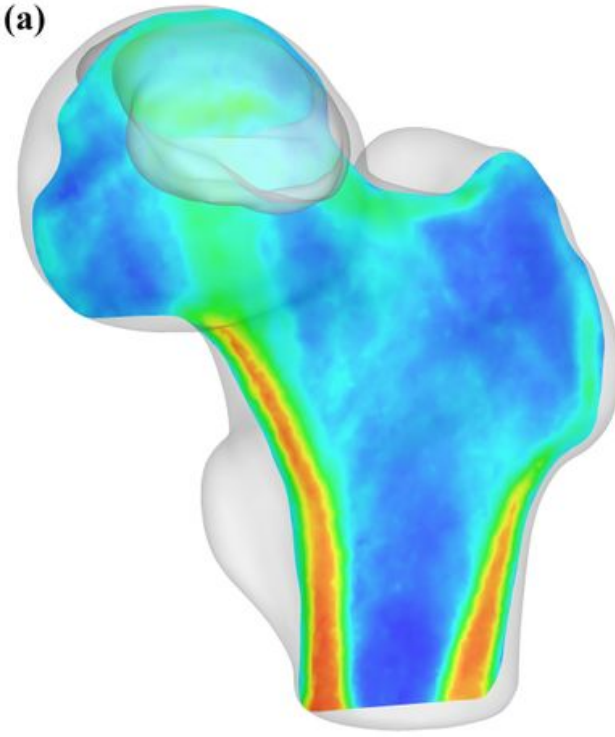

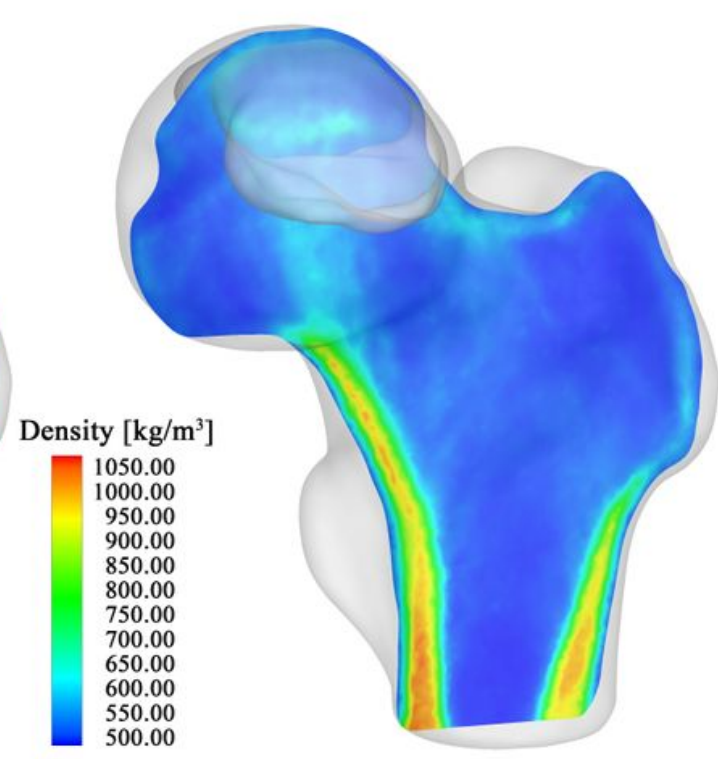

(b)

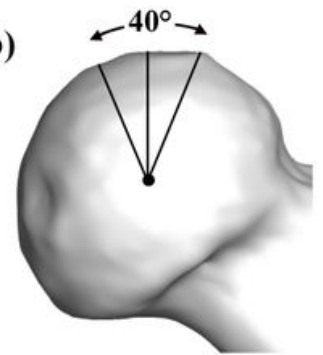

Young's

Modulus

[GPa]
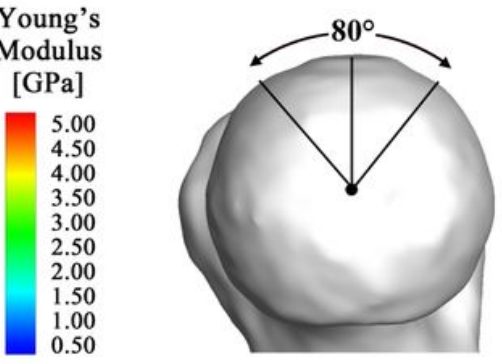

Figure 3

(a) shows the HU-derived density and Young's modulus distribution in one of the patient-specific models as a representative; (b) shows the force contact region of the superior femoral head 
(a) Pre-treatment

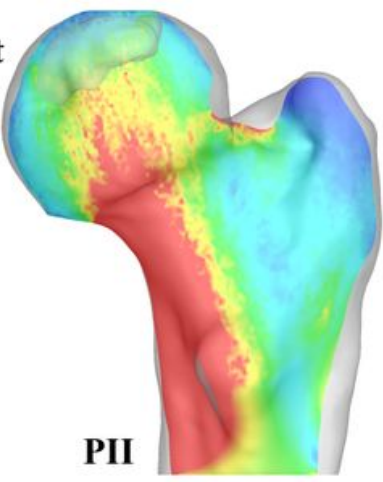

(b) Post-treatment

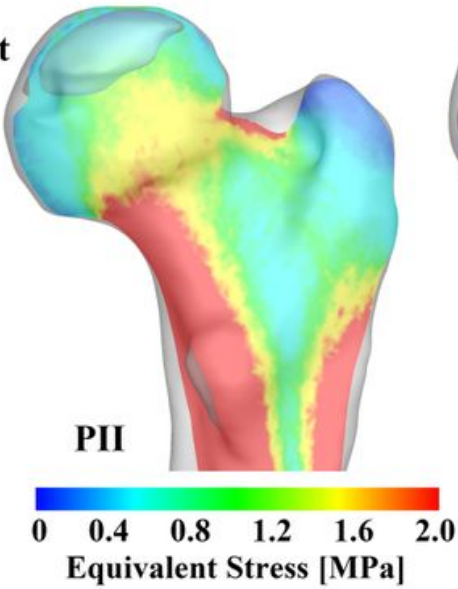

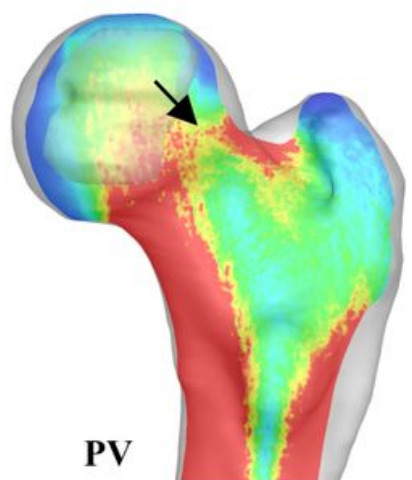

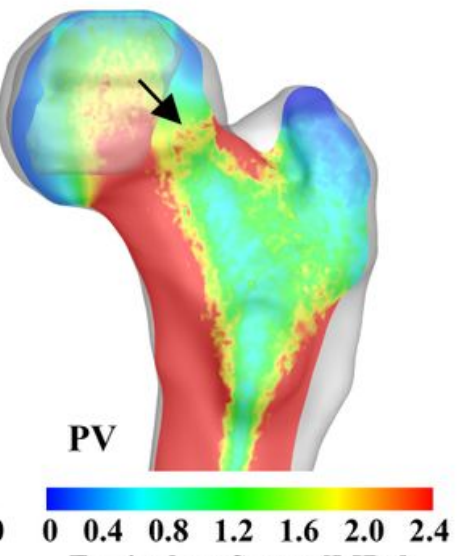

Equivalent Stress $[\mathrm{MPa}]$ (c) Healthy case

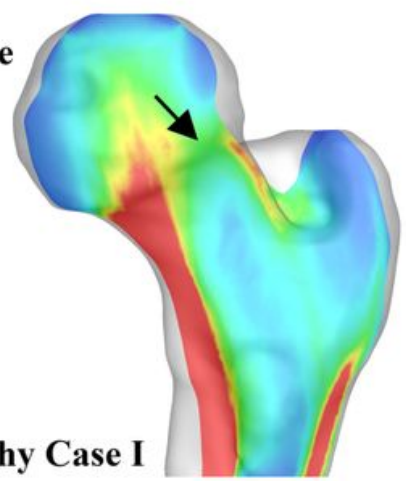

Healthy Case I

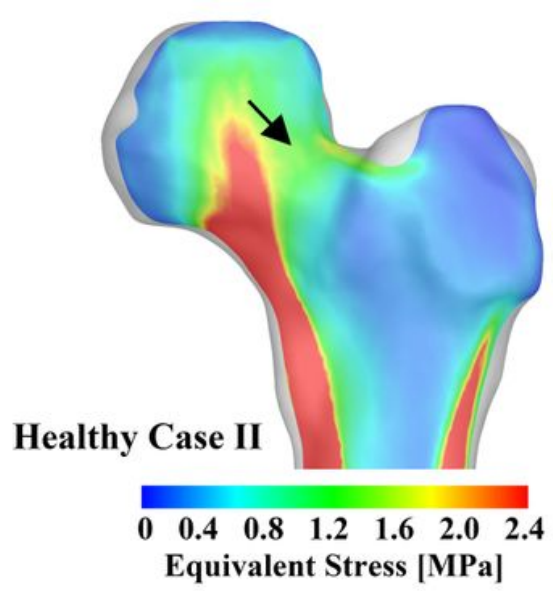

\section{Figure 4}

The stress distribution along the coronal slice of the proximal femur. (a) and (b) show the stress distribution in one of the uncollapsed and one of the collapsed models respectively as representatives; (c) displays the stress distribution in two healthy femurs;

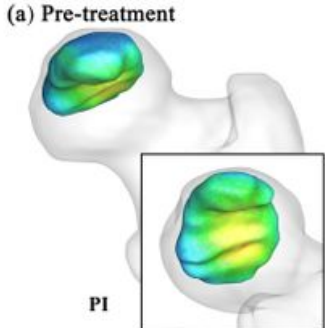

(b) Post-treatment

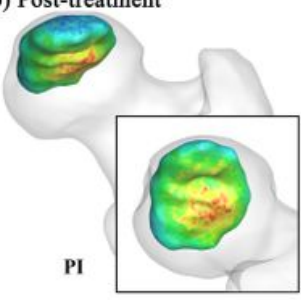

$\begin{array}{llllllll}0 & 0.3 & 0.6 & 0.9 & 1.2 & 1.5 & 1.8\end{array}$ Equivalent Stress [MPa]
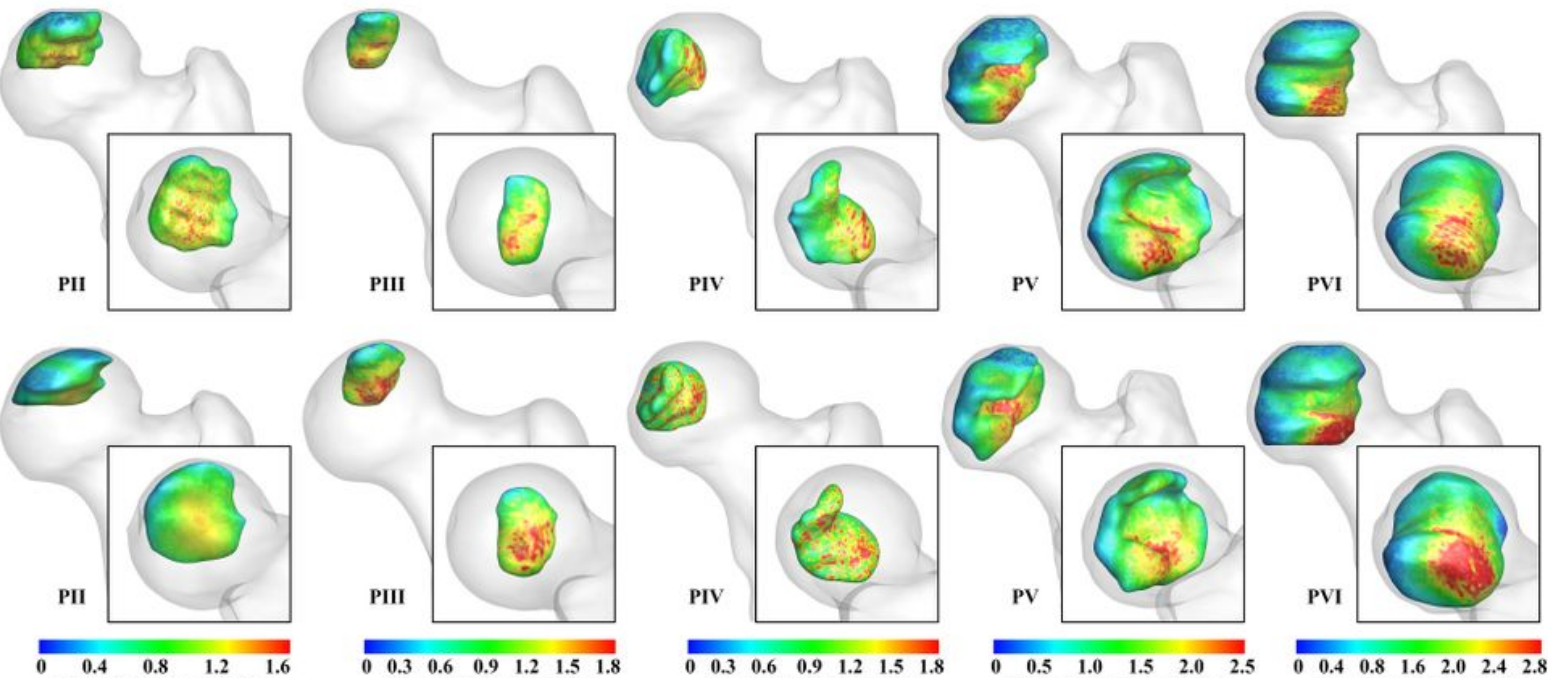

Equivalent Stress [MPa]
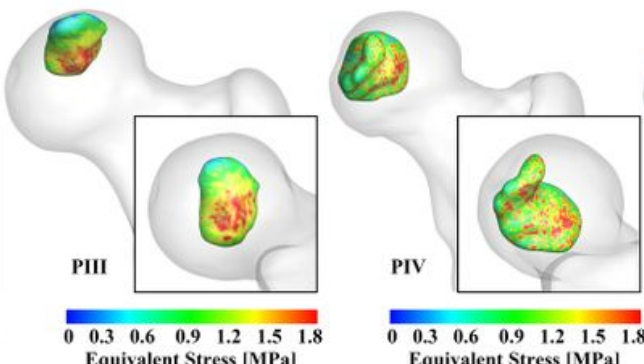

Equivalent Stress [MPa|
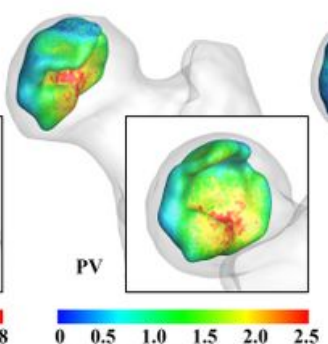

Equivalent Stress [MPa]

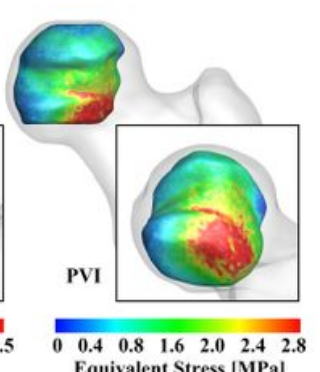

Figure 5 
The distribution of equivalent stress in the necrotic region. (a) and (b) display the stress distribution of the necrosis preand post- medical treatment, respectively. The magnified images in each subfigure show the stress distribution at the bottom of necrosis 